\title{
Laser-induced ablation of tantalum in a wide range of pulse durations
}

\author{
Steffen Mittelmann ${ }^{1}\left(\mathbb{D} \cdot\right.$ Jannis Oelmann ${ }^{2} \cdot$ Sebastijan Brezinsek ${ }^{2} \cdot$ Ding Wu $^{3} \cdot$ Hongbin Ding $^{3} \cdot$ Georg Pretzler $^{1}$
}

Received: 27 April 2020 / Accepted: 22 July 2020 / Published online: 5 August 2020

(c) The Author(s) 2020

\begin{abstract}
We present data and analysis of the laser-induced ablation of pure tantalum (Ta, $Z=73$ ). We have identified different physical regimes using a wide range of laser pulse durations. A comparison of the influence of strongly varying laser pulse parameters on high- $\mathrm{Z}$ materials is presented. The crater depth caused by three different laser systems of pulse duration $\Delta \tau_{1}=5 \mathrm{~ns}$ and wavelength $\lambda_{1}=1064 \mathrm{~nm}, \Delta \tau_{2}=35 \mathrm{ps}, \lambda_{2}=355 \mathrm{~nm}$ and $\Delta \tau_{3}=8.5 \mathrm{fs}, \lambda_{3}=790 \mathrm{~nm}$ are analyzed via confocal microscopy as a function of laser fluence and intensity. The minimum laser fluence needed for ablation, called threshold fluence, decreases with shorter pulse duration from $1.10 \mathrm{~J} / \mathrm{cm}^{2}$ for the nanosecond laser to $0.17 \mathrm{~J} / \mathrm{cm}^{2}$ for the femtosecond laser.
\end{abstract}

Keywords Pulsed laser ablation $\cdot$ High-Z material $\cdot$ Tantalum $\cdot$ Ablation threshold

\section{Introduction}

Short and ultra-short laser pulses are used in a wide spectrum of fields like material processing [1,2], nano-particle production [3,4] or in diagnostic applications [5]. Our purpose is to establish a reliable quantitative laser-induced breakdown spectroscopy (LIBS) diagnostic for deuterium retention analysis on inner walls of future fusion reactors as proposed by Huber et al. [6]. The goal is to provide a method that can be applied not only to present-day fusion facilities like EAST Tokamak in Hefei, China or W7-X Stellarator in Greifswald, Germany [7, 8], but also to future fusion devices like ITER [9].

The purpose of this paper is to compare the ablation behaviour of different laser systems that are promising candidates to perform first-wall diagnostic in this devices. In this context, laser-material interaction with laser pulses of different pulse durations must be compared and analyzed

Steffen Mittelmann

steffen.mittelmann@hhu.de

1 Institut für Laser- und Plasmaphysik, Heinrich-HeineUniversität Düsseldorf, 40225 Düsseldorf, Germany

2 Forschungszentrum Jülich GmbH, Institut für Energie- und Klimaforschung - Plasmaphysik, 52425 Jülich, Germany

3 Key Laboratory of Material Modification by Laser, Ion and Electron Beams, Chinese Ministry of Education, School of Physics, Dalian University of Technology, Dalian 116024, China concerning various effects. Early investigations by Chichkov et al. [10] present theoretical models compared to experimental results to describe a wide range of laser pulse duration interacting with solid surfaces. In general, these models predict a logarithmic dependence of ablation yield with respect to the laser pulse energy. However, for achieving a more detailed picture for the differences in ns-, ps-, and fs-ablation, the underlying individual mechanisms must be identified and discussed. When a ns-laser interacts with matter, it will generate plasma and heat it in the interaction region, leading to heat transport into un-illuminated regions of the target. The resulting rise in temperature leads to thermal material ablation including melting of the surface material. This process causes a large heat-affected zone (HAZ) that limits the depth resolution of information extracted from single pulse interaction. On the other hand, the interaction of the laser pulse with the plasma results in the so-called plasma shielding effect that limits the ablation rate at higher pulse energies [11].

When shorter laser pulses are employed, the influence of HAZ or plasma shielding decreases significantly [12], and these effects are not observed in ps- and even fs- single pulse ablation. Here the pulse duration is on the order of the thermal relaxation time (ps-laser) or much shorter (fs-laser). In this case, the high electro-magnetic fields excite the electrons in the skin depth (few nm behind the surface). Now the electron and lattice temperatures are decoupled and a breakdown that leads to ablation occurs on picosecond timescale [13]. That is why the predominant effect that drives the 
ablation depends on the non-linear response of the electrons to the strong laser field.

In $[14,15]$ and [16], the properties of the fs-ablation are discussed, with the shortest pulse durations in the order of 10-100 fs. The new effect in that case is that apparently, the ablated material has its origin exclusively in the area illuminated by the laser field (focus size, skin depth). Recent ablation studies using sub-10 fs pulses were performed on diverse dielectrics, e.g. fused silica or biological tissues [17, 18]. Note that sub-10 fs lasers require more complex facilities than ns- or ps-lasers for comparatively low pulse energy, that is why ps-lasers can be a promising compromise for our purpose and might be even used on a remote handling arm system. They deliver reasonable depth resolutions with a manageable experimental effort.

In this paper, we want to give an overview of the interactions by comparing the ablation thresholds and crater morphologies on the high- $Z$ material tantalum (Ta, $Z=73$ ) for three different laser systems. Compared to tungsten (W) or molybdenum (Mo), there are only few studies on laser interaction with this material $[19,20]$. With a similar mass as $\mathrm{W}$ but a much higher fuel content as seen by Hirai et al. [21] Ta can be used to test the methodology of fuel retention analysis. Separate experiments were carried out using identical Ta samples under comparable vacuum pressure with ns-, ps- and sub-10 fs laser pulses. The laser induced craters were analyzed postmortem and compared concerning morphology and depth.

\section{Experimental setup}

For the ablation threshold experiments, tantalum samples $(2 \mathrm{~mm} \times 50 \mathrm{~mm} \times 50 \mathrm{~mm})$ of $99.9 \%$ purity made by $H M W$ Hauner $\mathrm{GmbH} \& \mathrm{Co} . \mathrm{KG}$ were attached in vacuum chambers in a pressure range from $10^{-5}$ to $10^{-6}$ mbar. Here an unpolished sample was chosen to keep conditions similar for multiple laser shots on each position. The surface roughness of the samples was measured by confocal microscopy as $S_{a}=(0.7 \pm 0.3) \mu \mathrm{m}$. It is given as $S_{a}=\frac{1}{N} \sum_{k=1}^{N}\left|z_{k}-u\right|$ with the mean height $u$ over $N$ microscope positions. The root mean squared average is given as $R_{q}=(1.0 \pm 0.3) \mu \mathrm{m}$ and the surface exhibits an anisotropy with a ratio of 1.5 between parallel and perpendicular linear surface roughness. In the following, the three relevant experimental setups for the different laser systems are described in detail. An overview of the main laser parameters for the three systems is given in Table 1 . The laser pulse energy was verified by a thermal heat power meter measurements in each case.

\subsection{Nanosecond laser}

At Dalian University of Technology a Nd:YAG laser (Quantel Brilliant EaZy) of $5 \mathrm{~ns}$ duration, wavelength $1064 \mathrm{~nm}$ and energy of $280 \mathrm{~mJ}$ was used for the ablation experiment. The laser pulses with a close to flat-top beam profile were focussed into the vacuum chamber using a plano-convex fused silica lens of $30 \mathrm{~cm}$ focal length with an incidence angle of $0^{\circ}$ to the sample. The target was positioned in the converging laser beam, and two lens positions were used separated by $10 \mathrm{~mm}$. The laser spot sizes are given as $(410 \pm 3) \mu \mathrm{m}$ and $(720 \pm 3) \mu \mathrm{m}(5 \mathrm{~mm}$ and $15 \mathrm{~mm}$ from focal plane). To control the laser pulse energy the timing of the flash lamp that pumps the active laser medium and the Q-switch that releases the pulse from the cavity was changed. For pulse energies below $50 \mathrm{~mJ}$, different neutral density filters were applied in addition. 200 laser pulses with a repetition rate of $2 \mathrm{~Hz}$ were used for each crater.

\subsection{Picosecond laser}

The Nd: $\mathrm{VO}_{4}$ laser (EKSPLA PL2241) used at FZ Jülich provides laser pulses of $35 \mathrm{ps}$ duration and $46 \mathrm{~mJ}$ energy. We used a repetition rate of $1 \mathrm{~Hz}$ of the third harmonic $(\lambda=355 \mathrm{~nm})$. A plano-convex N-BK7 lens of $50 \mathrm{~cm}$ focal length focussed the transversal close to flat-top shaped laser beam into the vacuum chamber with $0^{\circ}$ incidence to the used sample. The spot size of the divergent laser beam at the sample surface was changed using three different lens positions. The laser spot diameters were determined as $(260 \pm 3) \mu \mathrm{m}$, $(620 \pm 3) \mu \mathrm{m}$ and $(960 \pm 3) \mu \mathrm{m}(1 \mathrm{~cm}, 2 \mathrm{~cm}$ and $3 \mathrm{~cm}$ out of focal plane) by a beam profile scan using a CMOS camera in the target plane. Again 200 laser pulses were used to create each crater. The pulse energy of the laser was controlled via the timing of the flash lamp that pumps the Nd:YAG rods within the cavity similar to the nanosecond laser. Again neutral density filters were used to further reduce the laser power down to the ablation threshold.

\subsection{Femtosecond laser}

The Phase-Stabilized Heine Laser (PHASER) at the Institute of Laser and Plasma Physics at Heinrich-Heine University in Düsseldorf provides laser pulses in the sub-10 fs pulse duration regime. Here an optimized commercial system (Femtopower Pro, Femtolasers $\mathrm{GmbH}$ ) was used in combination with a hollow fiber for spectral broadening. The Ti:Sa laser has pulse energies of $1 \mathrm{~mJ}$ with a repetition rate of $1 \mathrm{kHz}$ at a central wavelength of $790 \mathrm{~nm}$. Due to the ultrashort pulse characteristics the pulses exhibit a spectral bandwidth of $\Delta \lambda= \pm 150 \mathrm{~nm}$. A daily measurement by a SPIDER (Spectral Phase Interferometry for Direct Electric-field Reconstruction [22], VENTEON GmbH) is used to control the spectral phase and the pulse duration. The pulse energy in the experiment was controlled via a reflective attenuator 
consisting of gold mirrors of defined layer thickness. With this device the energy can be tuned in a range of four order of magnitude without changing any other optical parameter, as divergence or pulse duration. The 8.5 fs laser pulses with their spatial Gaussian profile were focussed by a $90^{\circ}$ off-axis parabolic mirror with an effective focal length of $127 \mathrm{~mm}$. The incidence angle onto the sample was $45^{\circ}$ and the laser is polarized parallel to the tilted surface. An optical diagnostic by an objective was used to determine the size of the focal spot diameter of $D_{0}=4 \mu \mathrm{m}$ (full width at $1 / e^{2}$ of maximum intensity). This every-day diagnostic was cross-checked by a beam waist measurement using a $2 \mathrm{~mm}$-scan with a profiler camera through the focal plane. The position of the sample along the optical path could be changed and fresh target position were set by a $x$-, $y$ - and $z$ - translation stage with $1 \mu \mathrm{m}$ accuracy. Craters were produced by 200 laser pulses at each sample position in a $2 \mathrm{~Hz}$ operation mode that can be achieved using a pulse-picking system within the laser. The sample was placed $300 \mu \mathrm{m}$ and $1000 \mu \mathrm{m}$ away from the focus in the divergent part of the laser beam. These sample positions go along with laser spot diameters of $(63 \pm 3) \mu \mathrm{m}$ and $(230 \pm 3) \mu \mathrm{m}$.

\section{Evaluation of the results}

First of all the different pulsed lasers need to be described by the same parameters. Here we focus on the laser fluence and intensity. For a flat-top beam profile the laser fluence, typically given in $\mathrm{J} / \mathrm{cm}^{2}$, is calculated from the pulse energy $E$ similar to [18] as

$F=\frac{4 E}{\pi D_{0}^{2}}$,

where $D_{0}$ is the beam diameter at $1 / e^{2}$ of the maximum intensity in the interaction plane. The angle of incidence $\Theta$ can be taken into account by multiplication with $\cos \Theta$. To compare this with a Gaussian shaped beam profile a factor 2 is included to take the smaller effective area into account. The intensity is given as $I=F / \Delta \tau$, with the pulse duration $\Delta \tau$ (FWHM of laser intensity in time). Note that $F$ is an integrated quantity and, therefore, gives a measure for the total effect induced by a laser pulse, whereas $I$ is a temporal quantity which describes momentary effects.

One aim of this investigation is to find the so called threshold fluence $F_{\text {th }}$ which is the minimum laser pulse energy per area required for material removal from the surface from a single laser pulse. On the other hand, also the threshold intensity $I_{\text {th }}$, the minimum laser power per area needed for ablation, is of interest when observing the influence of high electromagnetic fields. We want to have a look at both parameters because they become important in different physical regimes. The laser fluence has its relevance in the case of thermal processing of the used samples. Here we can refer to the process of ns-ablation or high repetition of ultra-short pulses on optical components. We refer to intensity when the ablation process is dominated by the basic electro-magnetic field interaction with the used material. Here single-shot ablation with ultra-short laser pulses are of interest.

From the crater depth variation with laser pulse energy, the threshold fluence can be retrieved by

$L=\beta^{-1} \ln \left(\frac{F}{F_{\text {th }}}\right)$,

where $L$ is the ablation depth per pulse called ablation rate [10]. A similar dependence can be observed with respect to a threshold intensity $I_{\text {th }}$ as

$L=\beta^{-1} \ln \left(\frac{I}{I_{\text {th }}}\right)$.

Note that $\beta^{-1}$ is a numerical fitting parameter that has the meaning of an effective penetration depth from the observed material. In the ps- to fs- pulse duration regime, this parameter can be set to the optical penetration depth $\alpha^{-1}$ as carried out in [23] for the case of a molybdenum target. The logarithmic dependence presented in Eqs. (2) and (3) has its origin in the assumption of a laser source term in the heat diffusion equation that transfers its energy into the material with an exponential decay constant $\beta^{-1}$. For the Ta sample, the optical penetration depth is calculated using

$\alpha^{-1}=\frac{\lambda_{0}}{4 \pi \cdot \kappa}$,

with the extinction coefficient $\kappa$ calculated in [24]. For the ns-laser $\beta^{-1}$ can be seen as a combination of optical and thermal penetration depth.

The crater depth has been evaluated via white light confocal microscopy (Micromeasure 2 from STIL) and the ablation rate is determined as the ratio of maximum crater depth and number of laser pulses at each position. We chose 200 laser pulses for each crater, because surface roughness and impurity effects are only effective for the first few shots, followed by a regime of linear crater depth increase [25]. Tani et al. found a lower ablation rate for the early laser pulses on the polished surface at low fluences with a fs-laser on copper. The resulting offset is neglected here due to the high surface roughness of the target in the first place. 


\section{Results and discussion}

To compare the different crater morphologies that are generated by laser pulses of different pulse durations. Figure 1 shows raw data from three craters in 2D false color plots
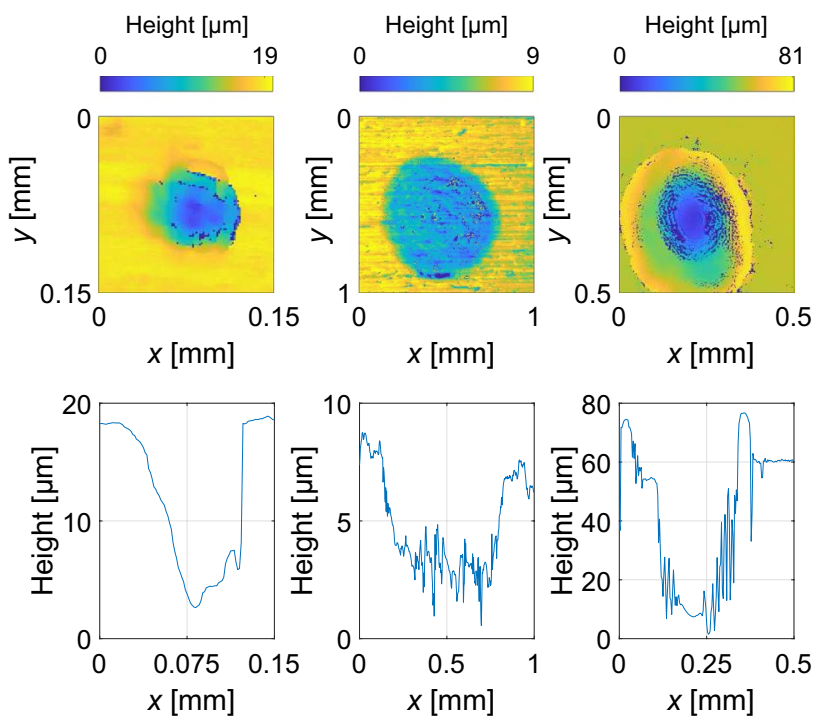

Fig. 1 Comparison of raw data from the different craters after the interaction with 200 laser pulses of pulse duration and fluence (left) $\Delta \tau=8.5 \mathrm{fs}, \quad F=2.4 \mathrm{~J} / \mathrm{cm}^{2}, \quad D_{0}=63 \mu \mathrm{m}, \quad$ (middle) $\Delta \tau=35 \mathrm{ps}$, $F=45 \mathrm{~J} / \mathrm{cm}^{2}, D_{0}=620 \mu \mathrm{m}$ and (right) $\Delta \tau=5 \mathrm{~ns}, F=24 \mathrm{~J} / \mathrm{cm}^{2}$, $D_{0}=410 \mu \mathrm{m}$ are shown. In the first row, the confocal microscope data in a false color plot and in the second row cross sections from the craters center are given

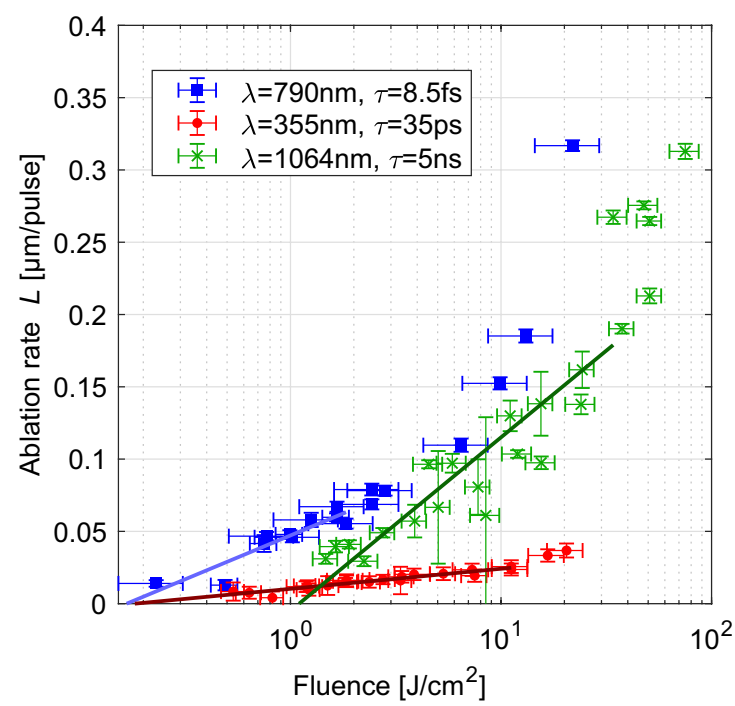

Fig. 2 (left) Ablation rate $L$ with respect to the used laser fluence $F$ and (right) ablation rate with respect to intensity with logarithmic $x$-axis are shown for three different laser systems. Here the filled and their cross section, respectively (averages of 11 curves). The morphology of these three craters reflects characteristic properties for the individual laser systems: The fs-craters on the left side exhibit an elliptical shape due to the $45^{\circ}$ incident angle onto the target. Around the ns-crater on the right side, a solidified corona of material can be observed.

The raw data were numerically smoothed where necessary, and ill-defined points were replaced. The depth is calculated by the difference of the mean surface height and a value from the craters center. Here 1000-20, 000 data points, depending on the resolution, are used. Due to the cone-like depth shape of the fs- and ns- craters, this value is given as the minimum of the measured height profile. The ps-laser generates cylinder like shapes in the center of the craters. That is why the depth value is calculated as a mean value from the craters center.

Figure 2 shows the measured ablation rate with respect to laser fluence and intensity. Table 1 gives the resulting threshold and effective penetration depth values from the numerical fitting process for the different laser systems with 17, 22 and 23 craters for the fs-, ps- and ns-laser, respectively. Here the ablation rate with respect to the laser fluence is plotted on a logarithmic $x$-axis shown in Fig. 2a. We observe smaller ablation rate with decreasing laser fluence. In Fig. $2 b$, the ablation rate is plotted with respect to the laser intensity on a logarithmic $x$-axis. The error bars correspond to the uncertainty of the laser pulse energy measurement caused by the power meter of $\pm 5 \%$. This is linked to the uncertainty of the beam diameter in the interaction plane of $\pm 3 \mu \mathrm{m}$. The uncertainty of the craters depth is caused by the surface roughness at each individual confocal microscope position.

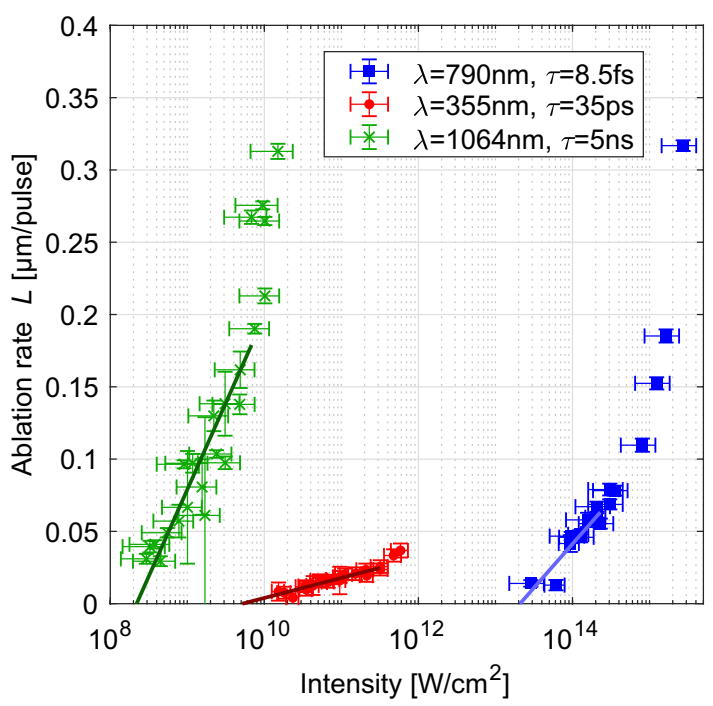

square (-) marker defines the femtosecond laser, filled circle $(\bullet)$ defines the picosecond laser and times $(X)$ defines the nanosecond laser measurement 
Table 1 Laser parameters for the ablation experiments from the three different systems and determined ablation threshold fluence $F_{\text {th }}$, intensity $I_{\text {th }}$ and effective penetration depth $\beta^{-1}$ compared to cal-

\begin{tabular}{lllllllll}
\hline$\Delta \tau$ & $E[\mathrm{~mJ}]$ & $\lambda_{0}[\mathrm{~nm}]$ & $D_{0}[\mu \mathrm{m}]$ & $\Delta z[\mathrm{~mm}]$ & $F_{\text {th }}\left[\mathrm{J} / \mathrm{cm}^{2}\right]$ & $I_{\mathrm{th}}\left[\mathrm{W} / \mathrm{cm}^{2}\right]$ & $\beta^{-1}[\mathrm{~nm}]$ & $\alpha^{-1}[\mathrm{~nm}]$ \\
\hline $5 \mathrm{~ns}$ & $1-270$ & 1064 & 410,720 & $-5,-15$ & $1.1 \pm 0.1$ & $(2.2 \pm 0.5) \cdot 10^{8}$ & $52 \pm 2$ & 13 \\
$35 \mathrm{ps}$ & $4-24$ & 355 & $260,620,960$ & $10,20,30$ & $0.18 \pm 0.10$ & $(5.2 \pm 3.0) \cdot 10^{9}$ & $6.0 \pm 0.3$ & 11 \\
$8.5 \mathrm{fs}$ & $0.1-0.5$ & 790 & 63,230 & $0.3,1$ & $0.17 \pm 0.05$ & $(2.1 \pm 0.5) \cdot 10^{13}$ & $26 \pm 1$ & 16 \\
\hline
\end{tabular}

The sample position relative to the focal plane $z_{0}$ is given as $\Delta z=z-z_{0}$

The pulse-to-pulse fluctuations of the laser pulse energy are ignored in each case, because of the high number of pulse repetitions (200) used for one crater. Using fits (solid lines) on the data given by Eqs. (2) and (3), threshold fluence and intensity result from the best fitting parameters. Graphically, this values can be observed as the roots and $\beta^{-1}$ as the slope of the fitting functions. Note that the used fitting function is only valid in a low fluence regime that depends on the pulse duration. For the fs-laser, the fit was made at fluences below $1 \mathrm{~J} / \mathrm{cm}^{2}$, for the ps-laser the limit was $10 \mathrm{~J} / \mathrm{cm}^{2}$ and within the ns-interaction $35 \mathrm{~J} / \mathrm{cm}^{2}$ turns out to be a valid limit. At this point, thermal penetration becomes more relevant and the slope of the logarithmic function rises.

To observe the influence of thermal and optical penetration during the interaction, we can compare the effective penetration depth $\beta^{-1}$ to the optical penetration depth $\alpha^{-1}$ from Eq. (4). The wavelength-dependent values are $16 \mathrm{~nm}$ for the fs-, $13 \mathrm{~nm}$ for the ns- and $11 \mathrm{~nm}$ for the ps-laser. Note that the wavelength dependence of the ablation process can be observed in the deviating slope of the ps-ablation and the fs-ablation curve. The resulting effective penetration depth of the ps- and fs-ablation differs by a factors less than 2 from the optical penetration depth $\alpha^{-1}$. For the ns-ablation, however, this factor is 4 , which indicates the high influence of thermal penetration that dominates this process. This result and the morphology comparison show the high thermal impact of ns-laser-matter interaction. While the fs- and ps-laser pulses produce craters with smooth edges, the nscraters exhibit a ring of solidified molten material higher than the surface around the hole. This expulsion of the melt lowers the precision, when the data from the ablation yield shell be used for information on the material content in the target as in our project. The timescale of the energy transfer between free electrons and the lattice is significantly longer than the pulse duration of these ultra-short laser pulses, which results in a considerably lower HAZ [26]. From the threshold analysis, we can observe a decreasing threshold fluence from $1.10 \mathrm{~J} / \mathrm{cm}^{2}$ for the ns-laser to $0.17 \mathrm{~J} / \mathrm{cm}^{2}$ for the fs-laser. The threshold intensity increases from $2.2 \cdot 10^{8}$ to $2.1 \cdot 10^{13} \mathrm{~W} / \mathrm{cm}^{2}$ with shorter pulse duration. Here the characteristics of the different interaction regimes is observed. The process is dominated by the high electro-magnetic culated optical penetration depth $\alpha^{-1}$ depending on used laser pulse duration $\Delta \tau$ and wavelength $\lambda_{0}$ are shown field in the ultra-short case (fs- and ps-ablation) and the minimum energy needed for ablation decreases. For fs- and ps-ablation, we observe the same threshold fluence value within the uncertainty. This can be related to the timescale of interaction that is in both cases before the breakdown in the material occurs. This phenomenon was also investigated by Genieys et al. [27], where the ablation threshold fluence for aluminum and tungsten was observed to be equal for sufficiently short laser pulses. Another work on this phenomenon was made by Gamaly et al. [28]. This observation can be expanded up to a ps-limit from our investigations on Ta. On the other hand, significantly higher intensity is needed with the fs-laser, namely up to five orders of magnitude more compared to the ns-laser. Other studies from the last years on Ta offer deviating results for $80 \mathrm{fs}$ [19] $\left(F_{\text {th }}=0.31 \mathrm{~J} / \mathrm{cm}^{2}\right)$ and $3 \mathrm{~ns}[20]\left(F_{\text {th }}=0.71 \mathrm{~J} / \mathrm{cm}^{2}\right)$ laser pulses. This deviation is caused by the fact that different wavelengths, pulse durations and ambient pressures are used. Another explanation can be the deviating behaviour of thin tantalum films deposited onto fused quartz samples in contrast to sheets of tantalum.

\section{Summary and outlook}

The described laser ablation on the high-Z material tantalum reveals the expected logarithmic dependence of ablation rate with respect to the laser pulse energy or power per area, respectively, for all used pulse durations and wavelengths. We are aware of the fact that the ablation threshold can vary for different polarization directions using a tilted target. Here further investigations are interesting. By observing the craters morphology and comparing the measured penetration depth to the optical penetration depth $\alpha^{-1}$ the high thermal impact of the ns-laser ablation is confirmed. By these concrete examples of laser ablation, the necessity of distinction between threshold fluence and intensity is pointed out. Moreover, by presenting these three pulse duration regimes on the same material, we get an idea of the best system for the diagnostic application. We can take advantage of the observed phenomena when generating LIBS signals from first-wall material in the fusion plasma devices. Here a high 
depth resolution is a relevant factor. It is shown that lowering the pulse duration offers the opportunity for high-resolution material analysis. Further work in this direction will bring reliable predictions of quantitative amount of the first-wall impurities.

Acknowledgements Open Access funding provided by Projekt DEAL. We want to thank for the opportunity to make confocal microscopy measurements in the MirrorLab at FZJ. Moreover, we gratefully acknowledge the financial funding by the Deutsche Forschungsgemeinschaft (No. 410415657), the National Natural Science Foundation of China (No. 11861131010) and the National Key R\&D Program of China (No.2017YFE0301304).

\section{Compliance with ethical standards}

Conflict of interest The authors declare that they have no conflict of interest.

Open Access This article is licensed under a Creative Commons Attribution 4.0 International License, which permits use, sharing, adaptation, distribution and reproduction in any medium or format, as long as you give appropriate credit to the original author(s) and the source, provide a link to the Creative Commons licence, and indicate if changes were made. The images or other third party material in this article are included in the article's Creative Commons licence, unless indicated otherwise in a credit line to the material. If material is not included in the article's Creative Commons licence and your intended use is not permitted by statutory regulation or exceeds the permitted use, you will need to obtain permission directly from the copyright holder. To view a copy of this licence, visit http://creativecommons.org/licenses/by/4.0/.

\section{References}

1. D. Sola, R. Cases, High-repetition-rate femtosecond laser processing of acrylic intra-ocular lenses. Polymers 12(1), 242 (2020)

2. C. Momma, B.N. Chichkov, S. Nolte, F. von Alvensleben, A. Tünnermann, H. Welling, B. Wellegehause, Short-pulse laser ablation of solid targets. Opt. Commun. 129(1-2), 134-142 (1996)

3. V.K. Pustovalov, A.N. Chumakov, Laser melting and evaporation of nanoparticles: a simplified model for estimations of threshold fluence. Opt. Laser Technol. 126, 106082 (2020)

4. S.S. Harilal, N. Farid, A. Hassanein, V.M. Kozhevin, Dynamics of femtosecond laser produced tungsten nanoparticle plumes. J. Appl. Phys. 114(20), 203302 (2013)

5. A. Huber, B. Schweer, V. Philipps, R. Leyte-Gonzales, N. Gierse, M. Zlobinski, G. Sergienko, Study of the feasibility of applying laser-induced breakdown spectroscopy for in-situ characterization of deposited layers in fusion devices. Phys. Scr. 2011(T145), $014028(2011)$

6. A. Huber, B. Schweer, V. Philipps, N. Gierse, M. Zlobinski, S. Brezinsek, U. Samm, Development of laser-based diagnostics for surface characterisation of wall components in fusion devices. Fusion Eng. Des. 86(6-8), 1336-1340 (2011)

7. C. Li, D. Zhao, Z. Hu, X. Wu, G.N. Luo, J. Hu, H. Ding, Characterization of deuterium retention and co-deposition of fuel with lithium on the divertor tile of EAST using laser induced breakdown spectroscopy. J. Nucl. Mater. 463, 915-918 (2015)
8. J. Oelmann, C. Li, S. Brezinsek, M. Rasinski, C.P. Dhard, R. König, C. Linsmeier, Depth resolved analysis of hydrogen in W7-X graphite components using laser-induced ablation-quadrupole mass spectrometry (LIA-QMS). Nucl. Mater. Energy 18, $153-158$ (2019)

9. J. Karhunen, A. Hakola, J. Likonen, A. Lissovski, M. Laan, P. Paris, J.E. Contributors, Applicability of LIBS for in situ monitoring of deposition and retention on the ITER-like wall of JET comparison to SIMS. J. Nucl. Mater. 463, 931-935 (2015)

10. B.N. Chichkov, C. Momma, S. Nolte, F. Von Alvensleben, A. Tünnermann, Femtosecond, picosecond and nanosecond laser ablation of solids. Appl. Phys. A 63(2), 109-115 (1996)

11. D. Bäuerle, Laser processing and chemistry (Springer Science \& Business Media, Berlin, 2000), pp. 221-257

12. R. Le Harzic, N. Huot, E. Audouard, C. Jonin, P. Laporte, S. Valette, R. Fortunier, Comparison of heat-affected zones due to nanosecond and femtosecond laser pulses using transmission electronic microscopy. Appl. Phys. Lett. 80(21), 3886-3888 (2002)

13. P.A. Zhokhov, A.M. Zheltikov, Optical breakdown of solids by few-cycle laser pulses. Sci. Rep. 8(1), 1-10 (2018)

14. S. Nolte, C. Momma, H. Jacobs, A. Tünnermann, B.N. Chichkov, B. Wellegehausen, H. Welling, Ablation of metals by ultrashort laser pulses. JOSA B 14(10), 2716-2722 (1997)

15. K.H. Leitz, B. Redlingshöfer, Y. Reg, A. Otto, M. Schmidt, Metal ablation with short and ultrashort laser pulses. Phys. Proc. 12, 230-238 (2011)

16. B.C. Stuart, M.D. Feit, S. Herman, A.M. Rubenchik, B.W. Shore, M.D. Perry, Nanosecond-to-femtosecond laser-induced breakdown in dielectrics. Phys. Rev. B 53(4), 1749 (1996)

17. M. Lebugle, N. Sanner, O. Utéza, M. Sentis, Guidelines for efficient direct ablation of dielectrics with single femtosecond pulses. Appl. Phys. A 114(1), 129-142 (2014)

18. L. Hoffart, P. Lassonde, F. Légaré, F. Vidal, N. Sanner, O. Utéza, I. Brunette, Surface ablation of corneal stroma with few-cycle laser pulses at $800 \mathrm{~nm}$. Opt. Express 19(1), 230-240 (2011)

19. E.V. Struleva, P.S. Komarov, S.I. Ashitkov, Characteristics of tantalum ablation under the impact of a femtosecond laser. High Temp. 56(5), 648-651 (2018)

20. L. Torrisi, F. Caridi, A. Picciotto, D. Margarone, A. Borrielli, Particle emission from tantalum plasma produced by $532 \mathrm{~nm}$ laser pulse ablation. J. Appl. Phys. 100(9), 093306 (2006)

21. T. Hirai, M. Rubel, V. Philipps, A. Huber, T. Tanabe, M. Wada, P. Wienhold, Testing of tungsten and tantalum limiters at the TEXTOR tokamak: material performance and deuterium retention. Phys. Scr. 2003(T103), 59 (2003)

22. C. Iaconis, I.A. Walmsley, Spectral phase interferometry for direct electric-field reconstruction of ultrashort optical pulses. Opt. Lett. 23(10), 792-794 (1998)

23. D. Zhao, N. Gierse, J. Oelmann, S. Brezinsek, M. Rasinski, Y. Liang, H. Ding, Investigation of laser ablation features of molybdenum bulk for picosecond laser-based techniques in fusion devices. Fusion Eng. Des. 151, 111379 (2020)

24. W.S. Werner, K. Glantschnig, C. Ambrosch-Draxl, Optical constants and inelastic electron-scattering data for 17 elemental metals. J. Phys. Chem. Ref. Data 38(4), 1013-1092 (2009)

25. S. Tani, Y. Kobayashi, Pulse-by-pulse depth profile measurement of femtosecond laser ablation on copper. Appl. Phys. A 124(3), 265 (2018)

26. A.H. Hamad, Effects of different laser pulse regimes (nanosecond, picosecond and femtosecond) on the ablation of materials for production of nanoparticles in liquid solution. High Energy Short Pulse Lasers 305-325 (2016) 
27. T. Genieys, M. Sentis, O. Utéza, Investigation of ultrashort laser excitation of aluminum and tungsten by reflectivity measurements. Appl. Phys. A 126(4), 1-14 (2020)

28. E.G. Gamaly, A.V. Rode, B. Luther-Davies, V.T. Tikhonchuk, Ablation of solids by femtosecond lasers: ablation mechanism and ablation thresholds for metals and dielectrics. Phys. Plasmas 9(3), 949-957 (2002)
Publisher's Note Springer Nature remains neutral with regard to jurisdictional claims in published maps and institutional affiliations. 